\title{
Ancillary detectors for the Mini-EUSO telescope: control software development and expected science
}

\author{
S. Turriziani*, M. Casolino and T. Ebisuzaki \\ for the JEM-EUSO Collaboration \\ RIKEN \\ E-mail: sara.turriziani@riken.jp, marco.casolino@riken.jp, \\ ebisu@postman.riken.jp
}

\begin{abstract}
Mini-EUSO will be devoted to study the UV light emission coming from Earth. It consists of a compact telescope with a large field of view ( $44 \times 44$ sq. deg.) based on an optical system with two Fresnel lenses and an UV filter. The light is focused into an array of photo-multipliers tubes and the resulting signal is converted to digital, processed and stored via on-board electronics subsystems. To complement the observations in other wavelengths, some ancillary cameras are present: a near infrared camera, a visible camera and an array of SiPM. The additional cameras will be used to monitor the observation conditions, and to obtain complementary information on several scientific topics that will be exploited with the main telescope, such as the physics of atmosphere, meteors, and strange quark matter. Here we review the expected science and present the general outline of the control software developed to stream data from the cameras together with the main instrument, in order to grab images in an automated way.
\end{abstract}

We dedicate this proceeding to Yoshiya Kawasaki and Jacek Karczmarczyk, who passed away in 2016.

35th International Cosmic Ray Conference - ICRC2017

10-20 July, 2017

Bexco, Busan, Korea

\footnotetext{
*Speaker.
} 


\section{The Mini-EUSO payload}

Mini-EUSO will be devoted to study the UV light emission coming from Earth (1). MiniEUSO is a small $\left(25 \times 35 \times 60 \mathrm{~cm}^{3}\right)$ light weight $(\sim 30 \mathrm{~kg})$ compact telescope based on an optical system with two Fresnel lenses (diameter: $25 \mathrm{~cm}$ ) and an UV filter. The light is focused into an array of photo-multipliers tubes (Photo Detector Module, PDM) and the resulting signal is converted to digital, processed and stored via on-board electronics subsystems. The telescope has a large field of view ( $44 \times 44$ sq. deg., which corresponds to $293 \times 293 \mathrm{~km}^{2}$ ). The PDM consists of an array of $6 \times 6$ Hamamatsu Multi-Anode Photo-Multiplier Tubes (MAPMTs). Each MAPMT has 64 pixels, so that the full PDM has 2304 pixels, achieving a spatial resolution of $6.11 \times 6.11 \mathrm{~km}^{2}$. A multiple level trigger algorithm has been implemented to recognize signals of interest, filtering out noise. Moreover, Mini-EUSO PDM's $2.5 \mu$ s time resolution allows for discriminating between fast and slow events, and following shower development of Ultra High Energy Cosmic Rays (UHECRs). A protective iris will be used in order to operate the PDM only during local night time on ISS, which corresponds to roughly $40 \%$ of an orbit. There will be no telemetry to monitor observations in real time directly from ground, and data transfer to Earth will occur periodically via exchange of data disk. Mini-EUSO will be placed inside the Zvezda module of the ISS to observe in nadir direction through the UV trasparent window. Mini-EUSO will produce the first high resolution UV map of the Earth in the $300-400 \mathrm{~nm}$ range, and these data will be publicly released. Moreover, MiniEUSO will observe atmospheric phenomena in the $\mathrm{ms}-\mu$ s range, such as lightning and Transient Luminous Events (TLEs). It will also record meteor data and search for nuclearities. It will also perform in-situ testing of the debris remediation system proposed by (2). Data will be recorded with $320 \mu$ s and $40 \mathrm{~ms}$ resolution for meteors and TLEs respectively. A more detailed description of Mini-EUSO science can be found in (3).

Approved by Italian and Russian Space agencies, it will fly to the International Space Station (ISS) from late 2017 to early 2018.
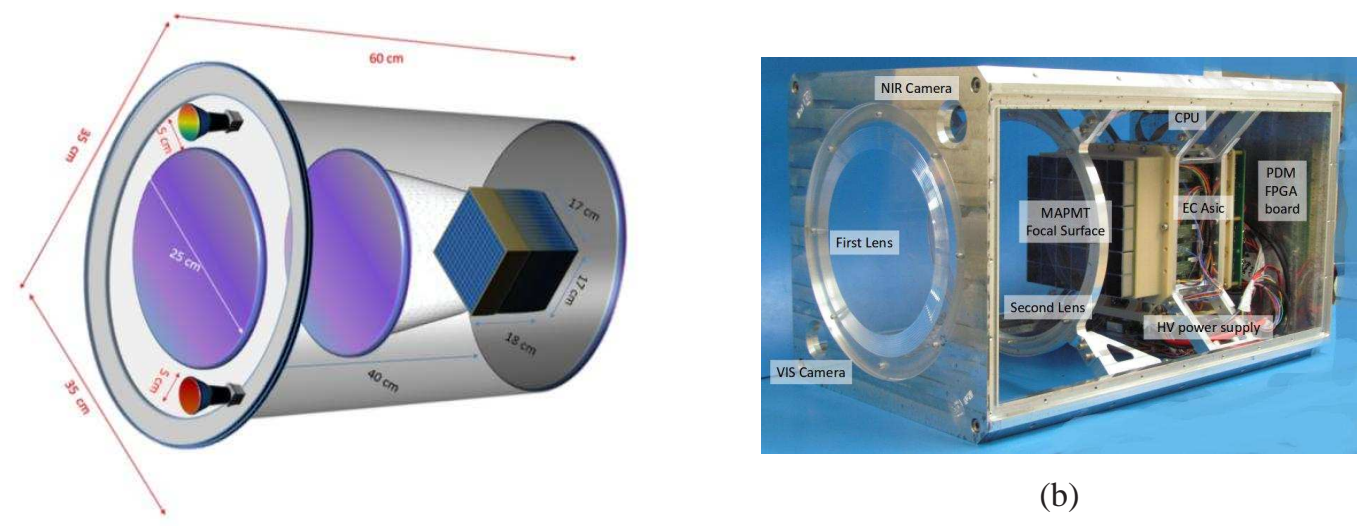

(b)

(a)

Figure 1: (a): a 3-D model of Mini-EUSO with all relevant dimensions shown; (b): a picture of Mini-EUSO EM with the different subsystems labelled. 
Table 1: Mini-EUSO Cameras Main Characteristics

\begin{tabular}{|l|c|c|}
\hline & NIR Camera & VIS Camera \\
\hline Camera Model & Chameleon 1.3 MP Mono USB 2.0 & Firefly MV 1.3 MP Color USB 2.0 \\
Sensor Name & Sony ICX445 & Sony IMX035 \\
Sensor type & CCD & CMOS \\
Sensor Size & $1 / 3^{\prime \prime}$ & $1 / 3 "$ \\
Readout Method & Global Shutter & Rolling Shutter \\
Maximum Resolution & $1296 \times 964$ & $1328 \times 1048$ \\
Standard Data Formats & $1280 \times 960,640 \times 480$ & $1280 \times 960,640 \times 480$ \\
Maximum Frame Rate & 8 -bit $1280 \times 960$ at $18 \mathrm{FPS}$ & 8 -bit $($ color $) 1280 \times 960$ at $15 \mathrm{FPS}$ \\
Pixel dimensions & $3.75 \mu \mathrm{m} \times 3.75 \mu \mathrm{m}$ & $3.63 \mu \mathrm{m} \times 3.63 \mu \mathrm{m}$ \\
Dimensions & $25.5 \mathrm{~mm} \times 44 \mathrm{~mm} \times 41 \mathrm{~mm}$ & $44 \mathrm{~mm} \times 34 \mathrm{~mm} \times 24.4 \mathrm{~mm}$ \\
Mass & $37 \mathrm{~g}$ & $37 \mathrm{~g}$ \\
Power Consuption & $2 \mathrm{~W}(\max )$ at $5 \mathrm{~V}$ & $<1 \mathrm{~W}$ \\
\hline
\end{tabular}

\subsection{Secondary Cameras Brief Overview}

The Mini-EUSO Additional Data Acquisition System (ADS) consists of two additional digital cameras mounted on the rim of the front end of the telescope (see Fig 1). The two cameras, one being sensitive in the infrared spectrum and the other in the visible range, will have the same operational time as the main telescope. Data from the cameras will help in the measurements of the emissions from Earth and the study of transient phenomena.

The visible camera (hereafter, VIS camera) was produced by FLIR Integrated Imaging Solutions Inc., formerly Point Grey Research. It is a 1/3" sensor, based on CMOS technology and equipped with an additional infrared cut-off filter at wavelegths above $750 \mathrm{~nm}$, making it sensitive in the $\sim 400 \mathrm{~nm}-750 \mathrm{~nm}$ wavelength range. The VIS camera sensor is a color filter array designed using a Bayer filter mosaic, i.e. some pixels are sensitive to Red, some to Blue, some to Green light. In other words, each pixel in the acquired image is either red, green or blue.

The near infrared camera (hereafter, NIR camera) was bought from Edmund Optics. The camera is actually a Black and White CCD Camera manufactured by FLIR to which $1 / 3$ " sensor a phosphorous coating was applied to allow for the conversion of near infrared photons to visibile range ones in order to get detected. The coating phosphorous absorption response ranges from $1500 \mathrm{~nm}$ to $1600 \mathrm{~nm}$, with two spectral peaks at $1512 \mathrm{~nm}$ and $1540 \mathrm{~nm}$.

Both cameras share the advantages to be light, less than $40 \mathrm{~g}$ each, and compact; moreover, they are low power devices. They are connected to the Mini-EUSO CPU via USB 2.0 connectors, and are separately powered via their GPIO connectors.

For some more technical details on the cameras, refer to Table 1.

Both cameras are equipped with a $8.5 \mathrm{~mm}$ fixed focal length C-mount lens, that yield a $31.8^{\circ}$ Field of View for a 1/3" sensor. Each lens has a weight of $54 \mathrm{~g}$, a length of $34.5 \mathrm{~mm}$, and no maximum working distance, extending performance out to infinity. Since the cameras support CS-mount lenses, CS to C Mount 5mm Spacer Adapters are added to obtain the correct focus.

Moreover, an $8 \times 8$ channels array of SiPM will be put on Mini-EUSO main focal surface. This photon-counting device uses multiple APD pixels operating in Geiger mode. It is an UV sensitive detector manufactured by Hamamatsu. It is a compact and thin device with high gain $\left(10^{5}\right.$ to $\left.10^{6}\right)$ and low bias voltage (around 60V). 


\section{Expected Science}

The additional cameras will be used to monitor the observation conditions, e.g. the presence of clouds and possibly infer some of their properties. Moreover, they will obtain complementary information on several scientific topics that will be explored with the main telescope, such as the physics of atmosphere, meteors, and strange quark matter.

Several thousands of meteors occur everyday in the Earth's atmosphere. However, a great amount of them happen during daylight, and they can be observed only if they are particularly bright: since Mini-EUSO operations will be performed during Earth nighttime from ISS we will observe only meteors happening during nighttime. Moreover, the brighter the fireball, the rarer the event will be: in fact, a general assumption is that the number of incident meteors increases by a factor of 2.5 per magnitude (4). However, the larger area covered from space will allow for measuring the influx and population index of the rare bright fireballs. Meteors are know to radiate strongly in the UV light, thus they are a perfect target for Mini-EUSO main camera. The calibration of the two secondary cameras is still ongoing, especially in order to estimate the limiting magnitude. Nonetheless, we would like to highlight that coupling the data from the Mini-EUSO main instrument with NIR and VIS measurements acquired with the ADS is a important to achieve a full picture of meteor physics. In fact, the emissions from meteors during their trajectory into the atmosphere have been studied intensively from UV to NIR, especially doing spectral analyses to determine their chemical composition (see e.g. 5). Both the vaporization of the meteroid and its heating effects on the surrounding atmosphere contribute to the emission from a meteor, that will result in a thermal continuum with superimposed various emission lines. For example, previous studies on the Leonid meteoric shower showed that their emission is characterized by relatively high temperatures (6), and that fast meteors coming from major showers exhibit flares in the late part of the trajectory. A typical meteor phenomenum is then explained as a hypervelocity flight in a free-molecule flow; however, this simple assumption may do not hold for really bright meteors, like fireball, since they penetrate deep into the atmosphere.

The observed brightness of a meteoroid in a given band will then depend on its kinetic energy. The simplest numerical entry model considers the interaction of air molecules with a meteoroid under varying flow conditions and quantifying the atmospheric and meteoroid response to this interaction. Moreover, the most elementary physical approach to the problem does not consider fragmentation, but considers only the ablation of a single body into the atmosphere. A good model is needed to estimate, for example, the original mass and velocity of the object on the basis of its observed light curve; however detailed modelling is quite complex as it requires considerations on hypersonic airflow, plasma physics, fragmentation dynamics, chemistry, non-equilibrium thermodynamics, radiative processes and materials science. Generally, the luminous intensity and the heat transfer coefficients in these models are the ones which give the greatest source of uncertainty (7). Collecting data for a meteor at different wavelengths is then important to infer a better estimation of the energy budget, and therefore to compare with theoretical models to constrain meteor mass. We underline here that not all the meteors observed with Mini-EUSO PDM will be detected with both the VIS and NIR cameras: this is due to both the smaller field of view of the two additional cameras with respect to Mini-EUSO main camera and to their limiting magnitudes as preliminary results from the calibration phase are showing that they are less sensitive than the PDM. However, 
we foresee that the VIS and NIR cameras will help the PDM in the characterization of the rarer bright fireballs that will happen to be in their field of view. Moreover, to date no observations of meteors have been done in the wavelegth range covered by the NIR camera, therefore even in case of no detection of meteors expected to be in its field of view, those observations can be used to put useful upper limits on the flux of meteors in that band, that can also be important to compare with theoretical predictions.

Nonetheless, cameras observation will be also exploited in searching for other objects burning in atmosphere, for example satellite and debris re-entries or possibly strangelet fragments, and in the monitoring of debris orbiting below the ISS. A satellite re-entry can be seen in fact as a large "artificial" meteor. Previous observations from the ground of rare bright re-entries showed interesting emissions signatures up to the NIR, characterazing then both the continuum and the spectral lines $(8 ; 9)$. The strong continuum is modelled as graybody emission coming from the hot ablated surface, whereas emission lines come from the bow shock and from trace metal impurities. For example, the Stardust capsule mostly showed continuum emission and air plasma emission, with weak lines of carbon, detected at $1069 \mathrm{~nm}$. The destructive ATV-1 reentry showed instead mostly AlO bands in the NIR.

On the other hand, strangelet meteorites or nuclearities, if exist, will be compact ultra-dense bodies made of very dense matter. In the case of a strangelet traversing the Earth's atmosphere the energy dissipation will be close to that of ordinary meteors, but ablation will be absent. Theoretical calculations also predict a bright meteor-like wake radiation, lasting $10^{-3}-10^{-1} s(10)$. Another important difference with respect to ordinary meteors would be in the velocity: although the existence of meteors of galactic velocities cannot completely be ruled out (11), strangelets can be distinguished in principle from ordinary meteors as meteors would disintegrate into the atmosphere, whereas stranglets would propagate down to the Earth's surface. Eventual detection of nuclearities or putting stronger upper limits on their flux is really important, since strange quark matter could contribute at least in part to the dark matter content of the Universe (12).

\section{Control Software Outline and Main characteristics}

The control software for the NIR and VIS cameras was developed in $\mathrm{C}++$ using FlyCapture Software Development Kit (SDK). FlyCapture SDK provides a common software interface to control and acquire images from FLIR USB 3.1, GigE, FireWire, and USB 2.0 cameras using the same API under 32- or 64-bit Windows or Ubuntu Linux. We used Flycapture2 (version 2.3.2.14) for 64-bit Linux. The software has been written in a way that there will be no need to recompile or change it between the Engineering Model and Flight Model or to make any different customization between the two.

The first step is the cameras initialization: the control software reads the desired parameters for each camera (i.e. BRIGHTNESS, FRAME RATE, etc) from a parameter file (hereafter, parfile). A two-parfiles-scheme was implemented for each camera, meaning that the software can choose between two different parfiles for the initalization of camera properties: they are, namely, a) the default parfile, which contains parameters validated and tested just before launch during the cameras calibration phase (on-going), and b) the current parfile, to use in case during the mission we 
would like to change any of the different parameters as maybe our pre-launch choices could be not the optimal ones for the actual observing conditions.

Before proceding to the initialization phase, the software: 1. checks for each camera if the current parfile exists; if not, it will update the camera register using the default parfile; 2 . however, even if the current parfile exists, some basic security tests are performed to validate it before it is used; 3. nonetheless, if any value for the parameters is not ok in this current parfile (e.g. the value is out of range), the camera register will be updated using the value listed in the default parfile instead. Parameters can be set totally independently for the two cameras: according to what is written in the parfile, the software will set on or off properties like BRIGHTNESS, FRAME RATE and AUTOEXPOSURE; then, it will set their values as listed in the parfile if they are set to on, and update also the values for the GAIN and the SHUTTER parameters. Exposure time for each camera is controlled by the combination of FRAME RATE and SHUTTER values, but only the SHUTTER parameter is used if the FRAME RATE is set to off in the parfile. Moreover, during initalization, the parameters SHARPNESS, SATURATION, IRIS, WHITE BALANCE, HUE, GAMMA, PAN, TILT, ZOOM, TRIGGER MODE, TRIGGER DELAY are always set to off if these parameters are supported by the camera. Note that each camera supports different parameters.

After both cameras have been successfully connected and initialized, they will start to acquire images at the same time, although not synchronized. Every single capture is recorded as a single image in 8-bit RAW format at $1280 \times 960$ for both cameras. A RAW format image contains no header or footer information, only pixel values are recorded. Each image is named following a DATE and TIME naming scheme. Moreover, a log file is also recorded.

The software (current version 3.5) is available on the GitHub platform. The array of SiPM will be operated by a different control software to be described elsewhere.

\section{Conclusions}

Mini-EUSO is a new large field of view ( $44 \times 44$ sq. deg.) telescope designed to study from the ISS the UV light emission coming from Earth (1). We described here the additional instruments that will contribute to complement the observations of the Mini-EUSO main UV camera, namely the NIR camera, the VIS camera and an array of SiPM. Control software has been developed to stream the NIR and the VIS cameras together with the main UV camera of Mini-EUSO so that they acquire images in an automated and independent way. The additional cameras are intended to monitor the observation conditions, e.g. the presence of clouds. Moreover, they will obtain complementary information on several scientific topics that will be exploited with the main telescope, such as the physics of atmosphere, meteors, and strange quark matter. Calibration of the cameras is ongoing to assess their performance, especially regarding their magnitude limits. At the end of this calibration phase, the final Mini-EUSO integration will take place and the cameras will be mounted on the rim on the front end of the main telescope. Mini-EUSO is expected to be launched from late 2017 to early 2018.

Acknowlegments This work was partially supported by the Italian Ministry of Foreign Affairs and International Cooperation, Italian Space Agency (ASI) contract 2016-1-U.0 "MINI-EUSO", State Space Corporation ROSCOSMOS, the Russian Foundation for Basic Research, grant \#1629-13065, and the Olle Engkvist Byggmästare Foundation. 


\section{References}

[1] M. Ricci, Mini-EUSO: a precursor mission to observe and study Atmosphere and Earth UV emission from the International Space Station, in 35th International Cosmic Ray Conference (ICRC2017), PoS (ICRC2017) 440, 2017.

[2] T. Ebisuzaki, M. N. Quinn, S. Wada, L. W. Piotrowski, Y. Takizawa, M. Casolino et al., Demonstration designs for the remediation of space debris from the International Space Station, Acta Astronautica 112 (July, 2015) 102-113.

[3] M. Casolino, A. Belov, M. Bertaina, G. Cambie', F. Capel, T. Ebisuzaki et al., Science of MINI-EUSO experiment on the International Space Station, in 35th International Cosmic Ray Conference (ICRC2017), PoS (ICRC2017) 369, 2017.

[4] F. Watson, Influences of Limiting Magnitude upon Meteor Frequency, Proceedings of the National Academy of Science 25 (May, 1939) 243-245.

[5] M. J. Taylor, P. Jenniskens, K. Nielsen and D. Pautet, First 0.961 .46 micron near-IR spectra of meteors, Advances in Space Research 39 (2007) 544-549.

[6] P. Jenniskens, S. J. Butow and M. Fonda, The 1999 Leonid Multi-Instrument Aircraft Campaign - An Early Review, Earth Moon and Planets 82 (2000) 1-26.

[7] M. D. Campbell-Brown and D. Koschny, Model of the ablation of faint meteors, AEA $4 \mathbf{4 1 8}$ (May, 2004) 751-758.

[8] M. J. Taylor, P. Jenniskens, I. Boyd, M. Wright, J. Olejniczak, G. McHarg et al., Near-Infrared Spectroscopy of Stardust Sample Return Capsule Entry: Detection of Carbon, Journal of Spacecraft and Rockets 47 (Nov., 2010) 878-883.

[9] J. B. Snively, M. J. Taylor and P. Jenniskens, Airborne imaging and NIR spectroscopy of the ESA ATV spacecraft re-entry: instrument design and preliminary data description, International Journal of Remote Sensing 32 (June, 2011) 3019-3027.

[10] M. S. Pshirkov, Prospects for strangelet detection with large-scale cosmic ray observatories, International Journal of Modern Physics D 25 (2016) 1650103, [1509.05553].

[11] V. L. Afanasiev, V. V. Kalenichenko and I. D. Karachentsev, Detection of an intergalactic meteor particle with the 6-m telescope, Astrophysical Bulletin 62 (Dec., 2007) 301-310, [0712.1571].

[12] A. de Rujula and S. L. Glashow, Nuclearites - A novel form of cosmic radiation, Nature 312 (Dec., 1984) 734-737. 